\title{
Screening per rilevare il maltrattamento in un ambiente ospedaliero pediatrico
}

\author{
Suresh S, Heineman E, Meyer L, et al. \\ Improved Detection of Child Maltreatment with Routine Screening in a Tertiary Care Pediatric Hospital \\ J Pediatr. 2021 Dec 17: S0022-3476(21)01185-9
}

\author{
Rubrica L'articolodelmese \\ a cura di Costantino Panza
}

\begin{abstract}
Il maltrattamento all'infanzia è una delle principali cause di morte e disabilità nei bambini. Il mancato riconoscimento dell'abuso nelle sue forme meno gravi può provocare abusi ripetuti e aumentare la morbilità e la mortalità; una percentuale significativa di bambini con lesioni da abuso è stata precedentemente valutata da un medico che non ha riconosciuto l'abuso. Per migliorare il rilevamento del maltrattamento in tutte le sue forme sono stati realizzati numerosi programmi di screening attraverso flowchart o checklist automatizzate o da eseguire in modo attivo da parte dell'operatore sanitario a contatto con il bambino. Il Pittsburgh Child Abuse Screening Tool è una checklist di cui è stata valutata l'efficacia in un setting ospedaliero con esiti incoraggianti anche se sono presenti problemi inerenti alla sensibilità e alla specificità della procedura.
\end{abstract}

Screening to detect Child Maltreatment in a Pediatric Hospital setting

Child maltreatment is one of the main causes of death and disability in children. Failure to recognize abuse in its less severe forms can lead to repeated abuse and increased morbidity and mortality. In fact, a significant percentage of children with abuse injuries had been previously evaluated by a physician who did not recognize the abuse. Many screening programs have been created using flowcharts or automated checklists to improve the detection of maltreatment in all its forms or to be actively carried out by the health worker in contact with the child. The Pittsburgh Child Abuse Screening Tool is a checklist whose effectiveness has been evaluated in a hospital setting with encouraging results even if there are problems inherent its sensitivity and specificity.

\section{Background}

Numerosi studi hanno rilevato che molte situazioni di maltrattamento infantile non sono riconosciute o non sono segnalate dai medici lasciando in tal modo il bambino in una situazione di rischio per lo sviluppo e per la incolumità fisica.

\section{Scopi}

Valutare l'impatto di uno screening di routine sul maltrattamento verso il bambino confrontandolo con un sistema di supporto decisionale incorporato nella cartella sanitaria elettronica già in uso in un ospedale pediatrico di terzo livello.

\section{Metodi}

Studio osservazionale prospettico. È stato progettato e realizzato un sistema di supporto alle decisioni cliniche sugli abusi nei bambini (CA-CDSS, Child Abuse Clinical Decision Support System) nel fascicolo sanitario elettronico ospedaliero consistente in trenta item (trigger); in caso di una situazione di sospetto maltrattamento fisico la compilazione della cartella elettronica con i dati della visita da parte del medico e/o infermiere fa scattare un alert che invita il sanitario a predisporre accertamenti per confermare o meno il sospetto di abuso. Parallelamente è stato elaborato un questionario (P-CAST, the Pittsburgh Child Abuse Screening Tool) da utilizzare come screening per il riconoscimento di sospette situazioni di maltrattamento basato su "Escape", un questionario già validato nei Paesi Bassi. Sono stati valutati 3 esiti primari: a) proporzione di pazienti con P-CAST positivo definito da almeno una risposta positiva, b) proporzione di pazienti con P-CAST positivo che non presentano trigger al CA-CSDD, d) proporzione di pazienti con P-CAST positivo segnalati al servizio sociale. Esiti secondari: valutazione della relazione tra etnia, stato socioeconomico e P-CAST positivo. Ogni esito è stato valutato per gruppi di età ( $<2$ anni; $2-12.99$ anni; $>$ 13 anni).

\section{Risultati}

Sono stati eseguiti 28.797 screening con P-CAST di cui 1.8\% (n. 512 ) è risultato positivo in bambini di età $<13$ anni e $1.6 \%$ positivo in bambini di età $\geq 13$ anni incapaci di comunicare verbalmente. Una metà delle visite con un P-CAST positivo (n. 215) ha anche attivato l'alert del CA-CDSS; l'altra metà con P-CAST positivo (n. 297) non ha avuto un riscontro nel sistema di supporto decisionale elettronico. Il 19\% dei pazienti con un P-CAST positivo è stato segnalato ai servizi di protezione dell'infanzia. Non era presente alcuna relazione tra etnia e la probabilità di un P-CAST positivo o tra etnia e la probabilità di una segnalazione ai servizi sociali.

\section{Conclusioni}

Lo screening di routine del maltrattamento fisico attraverso uno specifico questionario al quale il sanitario deve rispondere (Tabella 1) migliora l'identificazione di sospetti abusi in età pediatrica in un ospedale pediatrico rispetto ad un supporto decisionale incorporato nella cartella elettronica (CA-CDSS). 
Tabella 1. Confronto tra la checklist ESCAPE e P-CAST (tra parentesi l'ordine progressivo delle domande)

\begin{tabular}{|c|c|c|}
\hline Variabili & ESCAPE & P-CAST \\
\hline $\begin{array}{l}\text { Questioni sul racconto riportato } \\
\text { dal caregiver }\end{array}$ & $\begin{array}{l}\text { (D1) La storia è coerente? } \\
\text { (D3) L'insorgenza della lesione è compati- } \\
\text { bile con il livello di sviluppo del bambino? }\end{array}$ & $\begin{array}{l}\text { (D2) Sei preoccupato che la storia non possa essere coerente } \\
\text { con l'infortunio o la malattia? }\end{array}$ \\
\hline Ritardo nel cercare aiuto & $\begin{array}{l}\text { (D2) C'è stato un inutile ritardo nella ricer- } \\
\text { ca di assistenza medica? }\end{array}$ & $\begin{array}{l}\text { (D1) Per i bambini che si sono presentati per la valutazione di } \\
\text { un possibile incidente, c'è stato un possibile o sicuro ritardo } \\
\text { nel rivolgersi al medico data la gravità della/e lesione/i? }\end{array}$ \\
\hline Interazioni con i caregiver & $\begin{array}{l}\text { (D4) Il comportamento del bambino, dei } \\
\text { suoi caregiver e la loro interazione sono ap- } \\
\text { propriati? }\end{array}$ & $\begin{array}{l}\text { Domanda rimossa perché sulla base di precedenti discussio- } \\
\text { ni con infermieri e medici del dipartimento di emergenza in } \\
\text { merito a casi che non avevano segnalato al centro per la pro- } \\
\text { tezione dell'infanzia è presente la preoccupazione che questa } \\
\text { domanda potrebbe fornire al sanitario una giustificazione, } \\
\text { non basata su elementi di evidenza, per non segnalare quan- } \\
\text { do è stata osservata una interazione genitore-figlio conside- } \\
\text { rata adeguata. Questa domanda era anche non sensibile per } \\
\text { rilevare gli abusi nello studio di validazione del questionario } \\
\text { ESCAPE ma è stata inclusa perché di veloce compilazione. La } \\
\text { mancanza di sensibilità unita alle suddette preoccupazioni ha } \\
\text { portato alla rimozione di questa domanda. }\end{array}$ \\
\hline Esame fisico & $\begin{array}{l}\text { (D5) I risultati dell' esame clinico, dalla te- } \\
\text { sta ai piedi, sono conformi al racconto del } \\
\text { caregiver? }\end{array}$ & $\begin{array}{l}\text { (D3) È presente qualcuno dei seguenti risultati dell'esame fi- } \\
\text { sico? } \\
\text { - Età }<6 \text { mesi: qualsiasi livido, ustione, emorragia sottocon- } \\
\text { giuntivale o lesione del frenulo. } \\
\text { - Età } \geq 6 \text { mesi: a) lividi, ustioni o altri segni suggestivi di im- } \\
\text { patto con un oggetto; b) lividi su protuberanze non ossee/ } \\
\text { regioni protette (ad esempio tronco, genitali/glutei, braccia, } \\
\text { orecchie, collo); c) più lividi di quanto ci si aspetta di vedere } \\
\text { in un bambino attivo. }\end{array}$ \\
\hline Preoccupazioni per la sicurezza & $\begin{array}{l}\text { (D6) Ci sono altri segnali che ti fanno dubi- } \\
\text { tare sullo stato di protezione/sicurezza del } \\
\text { bambino o di altri membri della famiglia? }\end{array}$ & $\begin{array}{l}\text { (D4) Ci sono riscontri che potrebbero riflettere una scarsa su- } \\
\text { pervisione, cura, alimentazione o igiene? } \\
\text { (D5) Ci sono ulteriori commenti o dubbi relativi ad abusi o } \\
\text { negligenza sul bambino e/o spiegazioni aggiuntive per even- } \\
\text { tuali risposte "si" di cui sopra? }\end{array}$ \\
\hline
\end{tabular}

La mancanza di una relazione tra etnia e P-CAST positivo o segnalazione ai servizi sociali indica che lo screening sistematico del maltrattamento può mitigare le disparità etniche esistenti nell'identificazione e nella segnalazione di sospetti abusi nei bambini.

\section{Commento}

La maggior parte dei casi di maltrattamento ancora oggi non viene riconosciuta e segnalata. È presente una difficoltà nell'identificare il maltrattamento nel bambino da parte dei pediatri, e questa è una delle ragioni che portano a sottovalutare il numero delle vittime e dei casi mortali. In Europa sono stimati essere 850 ogni anno i casi di morte in età pediatrica imputabile ad abuso non riconosciuto [1]. Il maltrattamento è una condizione di cronicità e un precoce riconoscimento della situazione di rischio del bambino riduce il danno allo sviluppo e diminuisce il rischio di morte o di invalidità; prima della diagnosi molti bambini sono stati sottoposti a visite mediche senza che il professionista abbia riconosciuto o solo sospettato un maltrattamento dai sintomi o segni presenti. Per questo motivo sono stati progettati diversi programmi di screening che prevedono una flowchart o una checklist su particolari aspetti clinici quando si visita un bambino in un ambulatorio delle cure primarie o in un pronto soccorso. Lo scopo di questi interventi è di facilitare la diagnosi precoce e di promuovere gli interventi psicosociali nella speranza di interrompere la situazione di cronicità e favorire la protezione del bambino. Questi strumenti dovrebbero includere diverse componenti: sessioni di formazione specifica per gli operatori sanitari su come identificare i segni di trauma da maltrattamento, note automatizzate o checklist all'interno della cartella clinica elettronica per sollecitare un invio specialistico o a un team multidisciplinare di esperti [2]. Un investimento di sole 2 ore di e-learning per infermieri di pronto soccorso, incentrato sul riconoscimento del maltrattamento sui bambini con simulazioni di casi clinici e animazioni video ha mostrato un significativo miglioramento nel riconoscimento del maltrattamento [3], così come per il medico l'aver partecipato ad eventi formativi sul maltrattamento aumenta la probabilità di segnalare i casi sospetti $[4,5]$. Tuttavia una indagine alla quale hanno risposto pediatri di 148 ospedali in 29 paesi europei ha rilevato che solo il $28.6 \%$ degli intervistati ha utilizzato uno strumento di screening e solo il $31.8 \%$ considerava i fattori di rischio genitoriale; inoltre il $72 \%$ ha indicato la necessità di fare formazione. Complessivamente l'indagine ha 
riconosciuto una insufficienza di protocolli per il riconoscimento del maltrattamento all'infanzia nel 51.9\% dei pronto soccorso pediatrici rispetto ai protocolli esistenti nei servizi di emergenza degli adulti o misti adulti/bambini [6].

\section{Il problema degli screening per il maltrattamento}

In medicina gli interventi di screening sono progettati per identificare le condizioni che potrebbero in un momento futuro trasformarsi in malattie. Lo screening per il maltrattamento, tuttavia, non identifica un problema di salute e pertanto non produce una diagnosi. In realtà, gli strumenti di screening per il maltrattamento sui bambini codificano tipicamente diversi rischi e indicatori clinici di possibile o probabile maltrattamento (ad esempio il ritardo del caregiver nel rivolgersi al medico senza una spiegazione adeguata). In quanto tali, possono essere più correttamente indicati come strumenti che identificano potenziali maltrattamenti, o segni, sintomi e fattori di rischio che hanno una forte associazione con il maltrattamento e possono indurre l'operatore sanitario a considerare il maltrattamento come una possibile spiegazione della situazione clinica. La principale conseguenza di questa peculiarità dello screening per il maltrattamento è che difficilmente si può arrivare a misurare un'accuratezza diagnostica di tale metodica. Lo strumento Escape, una checklist di 6 voci che affronta i fattori di rischio che possono essere predittivi di maltrattamento (Tabella 1), ha dimostrato di migliorare il rilevamento di sospetti maltrattamenti rispetto a una popolazione di bambini non sottoposti a questo screening, con una con una sensitività di 0.80 e una specificità di 0.98 su oltre 18 mila visite al pronto soccorso [7]; Escape non è una checklist per la valutazione degli incidenti o dei traumi ed è utilizzato indipendentemente dal motivo della visita del paziente. Tuttavia, nella misurazione dell'efficacia di Escape, così come nella maggior parte di queste checklist, non si è tenuto in conto il rischio di bias di verifica, ossia il confronto con il gold standard per la rilevazione del rischio di maltrattamento, elemento necessario per considerare la reale probabilità di incorrere in casi falsi negativi e i falsi positivi. Considerando questo bias, il valore predittivo delle checklist si abbassa facendo aumentare sensibilmente i falsi positivi e negativi: un falso negativo indica un bambino che rimane in una situazione di grave rischio, che non riceve aiuto o che avrà un accesso ritardato ai servizi di protezione, mentre un falso positivo potrebbe portare tutta la famiglia a dover subire una indagine stressante e ingiustificata da parte dei servizi di protezione dell'infanzia [2]. Lo screening con P-CAST è risultato positivo nell' $1.8 \%$ dei casi (n. 512), ma sono stati segnalati ai servizi di protezione dell'infanzia solo 98 bambini (il 0.34\% complessivo dei casi giunti al pronto soccorso), senz'altro un miglioramento rispetto all'algoritmo automatizzato della cartella elettronica (42 casi in più rilevati e segnalati dal P-CAST e non rilevati dal CACDSS), ma ancora di difficile valutazione complessiva in carenza di verifiche prospettiche della popolazione esaminata.

\section{Non solo Checklist}

Cosa fare dunque? Queste checklist, se attuate senza una adeguata preparazione del personale sanitario e applicate come una rigida procedura di screening, possono comportare un numero elevato di bambini erroneamente sospettati o, all'opposto, persi. Tuttavia, se questi strumenti di screening sono inseriti in un più ampio protocollo di intervento possono ridurre la probabilità di non riconoscimento del maltrattamento. Una checklist inserita regolarmente in ogni visita pediatrica di pronto soccorso, associata all'adozione di un programma di formazione permanente sul maltrattamento all'infanzia di tutto il personale medico e paramedico che ha contatti con i bambini e, infine, l'istituzione in ogni ospedale di un equipe multidisciplinare specializzata nella valutazione delle situazioni a rischio migliora in modo significativo l'identificazione dei casi da portare all'attenzione dei servizi di protezione all'infanzia [8].

1. Hoedeman F, Puiman PJ, Smits AW, et al. Recognition of child maltreatment in emergency departments in Europe: Should we do better? PLoS One. 2021;16(2): e0246361. doi: 10.1371/journal.pone.0246361.

2. McTavish JR, Gonzalez A, Santesso N, et al. Identifying children exposed to maltreatment: a systematic review update. BMC Pediatr. 2020;20(1): 113. doi: 10.1186/s12887-020-2015-4.

3. Smeekens AE, Broekhuijsen-van Henten DM, Sittig JS, et al. Successful e-learning programme on the detection of child abuse in emergency departments: a randomised controlled trial. Arch Dis Child. 2011;96(4): 330-4. doi.org/10.1136/adc.2010.190801

4. Oral R, Blum KL, Johnson C. Fractures in young children: are physicians in the emergency department and orthopedic clinics adequately screening for possible abuse? Pediatr Emerg Care. 2003; 19(3): 148-53. doi: 10.1097/01.pec.0000081234.20228.33.

5. Flaherty EG, Sege R, Binns HJ, et al. Health care providers' experience reporting child abuse in the primary care setting. Pediatric Practice Research Group. Arch Pediatr Adolesc Med. 2000; 154(5): 489-93. doi: 10.1001/archpedi.154.5.489.

6. Hoedeman F, Puiman PJ, Smits AW, et al. Recognition of child maltreatment in emergency departments in Europe: Should we do better? PLoS One. 2021;16(2): e0246361. doi: 10.1371/journal.pone.0246361.

7. Louwers EC, Korfage IJ, Affourtit MJ, et al. Accuracy of a screening instrument to identify potential child abuse in emergency departments. Child Abuse Negl. 2014;38(7): 1275-81. doi: 10.1016/j.chiabu.2013.11.005.

8. Panza C, Berardi C, Apollonio MG, Paglino A. Maltrattamento all'infanzia. Manuale per gli operatori dell'area pediatrica. Il Pensiero Scientifico Editore 2020 